\title{
MiR-374b targets GATA3 to promote progression and development of glioblastoma via regulating SEMA3B
}

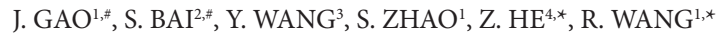 \\ ${ }^{1}$ Department of Image Diagnostic, The Affiliated Hospital of Inner Mongolia Medical University, Hohhot, China; ${ }^{2}$ Department of Dermatology, \\ The Affiliated Hospital of Inner Mongolia Medical University, Hohhot, China; ${ }^{3}$ Department of Clinical Laboratory, The Affiliated Hospital of \\ Inner Mongolia Medical University, Hohhot, China; ${ }^{4}$ Department of Neurosurgery, The Affiliated Hospital of Inner Mongolia Medical University, \\ Hohhot, China
}

*Correspondence: hezhanbiao1113@126.com; wangruijun1113@163.com

${ }^{*}$ Contributed equally to this work.

Received May 21, 2017 / Accepted July 22, 2017

\begin{abstract}
In the present study, a series of experiments were conducted to explore the function of miR-374b and the regulatory relationship among miR-374b, GATA3 and SEMA3B in glioma. MiR-374b mimics and inhibitors were employed to regulate miR-374b expression. Besides, qRT-PCR assay was used for detecting the expression level of miR-374b, GATA3 and SEMA3B mRNAs. To verify the targeting relationship between miR-374b and GATA3, dual luciferase analysis was utilized. Moreover, chromatin immunoprecipitation (ChIP) assay was performed to identify the correlation GATA3 with SEMA3B. Furthermore, si1-GATA3, si2-GATA3 and pc-GATA3 were used to regulate GATA3 expression and pc-SEMA3B served for dysregulation the SEMA3B. For assessing the significance of miR374b alone or in co-operation with GATA3 or SEMA3B on cell viability, migration and apoptosis, CCK-8, transwell and FCM assay were also performed. We found that overexpression of miR-374b, which was identified in glioma tissues and cell lines (U251, LN-299 and GOS-3) promoted cell migration and enhanced cell viability but inhibited apoptosis in this study. Furthermore, GATA3 contributed to increase the cell viability and migration and decrease the apoptosis targeted by miR-374b as evidenced by dual luciferase assay. Moreover, GATA3 binding to the promoter of SEMA3B, involved in regulating SEMA3B, was revealed. Further, a series of studies demonstrated that miR-374b targeted GATA3 regulating SEMA3B and resulted in elevation of cell viability and migration but suppressed the apoptosis. However, the promotion effects of miR-374 in glioma process were reversed by co-transfecting pc-GATA3 or pc-SEMA3B. In conclusion, miR-374b promotes glioma process in vitro through suppressing SEMA3B via targeting GATA3. The result of this study provides an important clue to the optimal treatment schedule for glioblastoma.
\end{abstract}

Key words: miR-374b, glioma, U251, GATA3, SEMA3B

Glioma is the most common brain tumor worldwide with the second highest mortality rate among intracranial tumors [1] and incidence up to 3.2 per 100000 in the United States [2]. Over the past few decades, many researches uncovering new and more effective therapies for glioma were conducted along with the improvement in surgical resection, radiotherapy and chemotherapy, but overall prognosis of patients with glioma is still unfavorable and dismal [3]. In addition, tumor cells usually generated chemoradiotherapy resistance, which was the reason of limited treatment and poor response [4]. Moreover, according to report, the most malignant glioma is glioblastoma multiforme [5]. Therefore, it is vital to explore a more comprehensive insight into molecular mechanism of glioma cell proliferation and metastasis and to find more appropriate therapeutic target.
MicroRNAs (miRNAs) are one kind of non-coding RNAs which play the crucial roles in various diseases, including glioma [6] cancer, ischemia-reperfusion injury in lung [7] or myocardium [8]. Because of their significance in cancers, they have been proposed by numerous investigations as novel biomarkers for many cancer diagnosis and treatment in recent years [9]. Approximately 50\% miRNAs expression level is associated with tumorigenesis or angiogenesis through influencing the expression of oncogenes or tumor suppressors [10]. The inhibition of miR-603 can repress glioma cell proliferation via elevating WIF1 and CTNNBIP1 to influence Wnt/ $\beta$-catenin pathway [11]. MiR-24-3p and miR-27a-3p cooperating with MXI1 (a member of mad family), a tumor suppressor, promote glioma progression and development [12]. These reports reveal that some miRNA expression in 
glioma accelerates its progression. MiR-374b, a novel kind of miRNAs abnormally expressed in gastric cancer, shows significant upregulation that promotes tumor cell invasion and metastasis [13], which elucidates that miR-374b may be oncogene in some cancers. Therefore, the research should be focused on miR-374b-5p role in glioma, to get more comprehensive insight into the diagnosis and therapy for this type of cancer.

GATA binding protein 3 (GATA3), one kind of transcription factor, functions as a vital regulator in differentiation of the luminal cell fate [14]. Besides, it is demonstrated that GATA3 is significantly restrained during breast cancer progression [15], indicating its possible tumor suppressor role as transcription factor. Thus, silencing GATA3 contributes to progression and development of various cancers including breast cancer [16], glioblastoma [17], prostate cancer [18] and bladder cancer [19], which shows the significance and therapeutic potential of GATA3 in treatment in multiple cancers. However, investigation on the effects of GATA3 in glioma is rare and should be taken seriously.

Semaphorin 3B (SEMA3B), located at 3p21.3, encodes a tumor suppressor protein for multiple cancers [20-22]. The mutation or promoter methylation of SEMAB3 is always associated with pathogenesis of lung cancer [23]. According to previous study, SEMA3B exerts its function via VEGF-A, VEGF-B and placental growth factor (PIGF) that are members of the vascular endothelial growth factor (VEGF) family [24-26]. Moreover, the high expression of GATA3 as well as the changes in SEMA3B contributed to suppress breast cancer progression and metastasis [27]. Meanwhile, gene expression profiling in glioblastoma elucidates SEMA3B inhibition [28]. Karayan et al. demonstrated the function of SEMA3B, whose downexpression was correlated with poor survival, as inhibitor of tumor progression via regulating VEGF ability [29]. But there are few reports about the regulatory relationship between GATA3 and SEMA3B in glioma.

In the present study, we assumed that miR-374b targeted GATA3 to suppress transcription of SEMA3B, which contributed to promote pathogenesis of glioblastoma. GATA3 functioning as transcription factor for SEMA3B in vitro was uncovered, and this investigation testified whether miR-374b contributed to cancer cell proliferation and migration via GATA3/SEMA3B.

\section{Materials and methods}

Human tissue samples. All human glioma tissues were provided by patients from The Affiliated Hospital of Inner Mongolia Medical University, Hohhot, China, who all accepted standard surgical operations. Besides, patients with traumatic brain injury underwent decompressive craniectomy and their healthy grain tissues were obtained. Liquid nitrogen was utilized to freeze the collected samples until analysis. In the present study, all patients gave informed consent before the study, and Inner Mongolia Medical University approved all the experiments conducted.

Cell culture. Human glioma cell line, U251, and human embryonic kidney cell line, 293T, were purchased from BeNa Culture Collection located at Beijing, China, and were maintained in Dulbecco's modified Eagle's medium (DMEM) containing 10\% fetal bovine serum (FBS). Besides, $100 \mathrm{U} / \mathrm{ml}$ penicillin and $100 \mathrm{ng} / \mathrm{ml}$ streptomycin were added into DMEM before incubating cells. However, for culturing $293 \mathrm{~T}$ cells, $2 \mathrm{mmol} / \mathrm{ml}$ glutamine was additionally added into DMEM. All cells were incubated at $37^{\circ} \mathrm{C}$ and $5 \% \mathrm{CO}_{2}$.

Quantitative reverse transcriptase PCR (qRT-PCR). TRIzol reagent from Invitrogen (Carlsbad, CA, USA) was employed based on the instructions of producer to extract total RNAs that were then transcribed into cDNA with PrimeScript RT Reagent Kit from Invitrogen. In the study, the primers used were as follows: GATA3 forward primer, 5'-CAGCCTTCGCTTGGGCTTAAT-3'; GATA3 reverse primer, 5'-GATGGCAGGCTCAGTGATGTC-3'; SEMA3B forward primer, 5'-CCTCAACCTGGACAACATCAGC-3'; SEMA3B reverse primer, 5'CGAAGTTCATGCACTCAGTACCAA T-3'; GAPDH forward primer, 5'-CCACCCATGGCAAATTCCATGGCA-3'; GAPDH reverse primer, 5'-TCTAGACGGCAGGTCAGGTCCACC-3'. In the present study, qRT-PCR was performed in $15 \mu \mathrm{l}$ system with SYBR Premix DimerEraser (Invitrogen) on a $7900 \mathrm{HT}$ system. Moreover, GADPH, the housekeeping gene, was considered as internal control and all primers were purchased from Invitrogen.

Western blot. $1 \times$ lysis buffer from Cell Signal Technology (CA, USA) was used to obtain cell extracts after cells washed twice with pre-cold PBS buffer. BCA assay was performed for determining the protein concentration. Afterwards, $50 \mu \mathrm{g}$ proteins were separated on $12 \%$ SDS/PAGE gels followed by transfer onto nitrocellulose membranes. $5 \%$ skimmed milk was added into TBS containing $0.1 \%$ Tween- 20 and the mixture was well mixed for blocking the membranes for $1 \mathrm{~h}$. Next, the membranes were incubated with primary antibodies overnight at $4{ }^{\circ} \mathrm{C}$ and washed with TBST. Further, secondary antibodies were employed. An enhanced chemiluminescence system from DuPont (Boston, MA) was utilized to visualize bound antibodies. The densitometric analysis was carried on with ImageJ software. All antibodies were purchased from Abcam (Cambridge, MA, USA): primary antibodies - rabbit anti-GATA3 (1:1000, ab106625) and rabbit anti-SEMA3B (1:5000, ab48197), and secondary antibody - horseradish peroxidase-labeled mice anti-rabbitGAPH (1:1000, ab8245).

Cell transfection. Cell were incubated in DMEM medium to $60 \%$ confluency, the GATA3 expression level was attenuated by its specific small interference RNA (siRNA), si1-GATA3 or si2-GATA3 transfection, and enhanced by pc-DNA3.1-GATA3 (named as pc-GATA3). Besides, aiming to obtain the changes in miR-374b expression, miR-374b mimics (named as mimics) and inhibitors (named as inhibi- 
tors) were employed. Moreover, the SEMA3B sequence was cloned into pc-DNA3.1 plasmids as pc-SEMA3B to up-regulate SEMA3B expression. To explore the correlation between miR-374b and GATA3, mimics + pc-GATA3 and inhibitors + si-GATA3 were transfected into cells. For the relationship of miR-374b and SEMA3B, mimics + pc-SEMA3B transfection was performed. In the present study, the scrambled miR-374b sequence was considered as control group for mimics and inhibitors, and the blank pc-DNA3.1 plasmids and scrambled siRNA sequence for overexpression plasmids and knocked down sequence. All sequences and plasmids were obtained from Thermo Fisher Scientific (Waltham, MA, USA).

Dual luciferase assay. Aiming to detect the regulatory relationship between miR-374b and GATA3, the luciferase reporter recombining the 3' UTR of the human wild type GATA gene (GATA3-wt) or mutant type GATA3 (GATA3mut) was purchased from RiboBio Company (Guangzhou, China). The reporter plasmids and internal control plasmids were both transfected into $293 \mathrm{~T}$ cells along with miR-374b mimics or the scrambled sequence $(100 \mathrm{pM})$. Furthermore, Dual-Luciferase ${ }^{\bowtie}$ Reporter Assay kit from Promega (Madison, WI, USA) was utilized to determine the luciferase signal. Moreover, the Turner Designs TD-20/20 was performed to detect the GATA3 promoter activation.

ChiP assay. For getting the comprehensive insight on whether GATA3 binding to SEMA3B promoter exerts its regulatory effects, chromatin immunoprecipitation (ChIP) assay was performed with MAGnify Chromatin Immunoprecipitation System (Invitrogen). Si1-GATA3, si2-GATA3 and NC group cells were prepared for ChIP assay and then crossed-linked by $1.2 \%$ formaldehyde for $10 \mathrm{~min}$. Afterwards, the chromatin was sonicated to achieve 200-1000 bp. Then, the antibodies for GATA3 or IgG (positive control) were utilized to obtain antigen-antibody complex, which were collected by protein A agarose beads from Invitrogen. These non-specific binding proteins were washed with buffer. To obtain the input \% of SEMA3B, PCR reaction was performed. In this study, Cell Signaling Technology (Danvers, MA, USA) provided all used antibodies and the primer sequences for SEMA3B promoter, Set \#1: forward primer 5'-AGACAGGTATGACCGTGACC-3' and reverse primer 5'-AGCTGTCTTGTGCTTGGGAAT-3'; Set \#2: forward primer 5'-CAGACCTCATGGGACGAGAC-3' and reverse primer 5'-TGGCTAGCTGTCTTGTGCTT-3', were obtained from Thermo Fisher Scientific.

Cell viability assay. MTT (thiazolyl blue) assay was conducted for assessing cell viability. First, single cell suspensions were collected using trypsin. 96-well-plates were used to incubate cells at a density of $1 \times 10^{3}$ cells/well for $48 \mathrm{~h}$ at $37^{\circ} \mathrm{C}$. Ten $\mu \mathrm{l} \mathrm{MTT}(5 \mathrm{mg} / \mathrm{ml}$ ) from Sigma-Aldrich (St. Louis, MO, USA) stock solution was added into each well containing $0.1 \mathrm{ml}$ medium, which was then incubated for $4 \mathrm{~h}$ at $37^{\circ} \mathrm{C}$, followed by adding $100 \mu \mathrm{l}$ DMSO for $5 \mathrm{~min}$ at room temperature to dissolve crystals. Lastly, to determine cell viability, the absorbance at a wavelength of $570 \mathrm{~nm}$ was measured.

Apoptosis assay. Annexin V-FITC Apoptosis Detection Kit (Invitrogen) was employed to quantify apoptotic cells based on the manufacturer's protocol. After transfection the cells were collected for staining with $5 \mu \mathrm{l}$ Annexin $\mathrm{V}$-FITC along with $10 \mu \mathrm{l} 20 \mu \mathrm{g} / \mathrm{ml}$ propidium iodide (PI). These samples were then incubated at dark for $15 \mathrm{~min}$. The apoptotic cells were detected by a FACScan flow cytometer.

Transwell analysis. Cell migration ability was determined with transwell analysis. In this study, a transwell chambers with $5.0 \mu \mathrm{m}$ pore size and $6.5 \mathrm{~mm}$ diameter polycarbonate filter were used to culture $1.5 \times 10^{5}$ cells. Besides, $100 \mu \mathrm{l}$ serum-free medium were added into upper chamber and the lower chamber was filled with $600 \mu \mathrm{l}$ medium with $10 \%$ FBS. $36 \mathrm{~h}$ after incubating at $37^{\circ} \mathrm{C}$ and $5 \% \mathrm{CO}_{2}$, the migrating cells were fixed and then stained by $0.5 \%$ crystal violet. Cell numbers were computed under a light microscope.

Statistical analysis. Student's t-test was conducted to assess the difference between two groups. In the present study, all data are presented as the mean \pm standard deviation (mean $\pm \mathrm{SD}$ ). Moreover, $\mathrm{p}<0.05$ was statistically significant. All experiments in this study were performed three times.

\section{Results}

High expression of miR-374b in glioma tissues and cell lines. We first examined the expression of miR-374b in glioma tissues and cell lines (U251, LN-229 and GOS-3) in comparison with healthy tissues and human astrocytes NHA. qRT-PCR results suggested that miR-374b was significantly upregulated in glioma tissues relative to healthy tissues (Figure 1A) and the expression of miR-374b in glioma cell lines was higher than in normal human astrocytes NHA (Figure 1B). Besides, the overexpression of $\mathrm{miR}-374 \mathrm{~b}$ in U251 was more obvious than in other glioma cell lines, so U251 was chosen for subsequent experiments.

Dysregulation of miR-374b contributed to influence the cell physiological state. To further investigate the miR-374b on glioma, miR-374b mimics and inhibitors transfections were employed to enhance and silence miR-374b expression. Scrambled miR-374b sequence was considered as control group (NC). qRT-PCR consequence demonstrated that miR-374b expression in mimics group was promoted compared with NC group, but the expression in inhibitors group was significantly inhibited, indicating the success of transfection (Figure 2A). Subsequently, CCK-8 assay was performed to determine cell viability. As shown in Figure $2 \mathrm{~B}$, cells in mimics group displayed remarkably stronger viability compared with NC group, and cell viability in inhibitors was significantly suppressed as expected. Besides, cell apoptosis ratio was investigated using FCM analysis. The results suggested that overexpression of miR-374b contributed to lower cell apoptosis rate and as we expect, silencing miR-374b resulted in higher one compared with NC group 
A

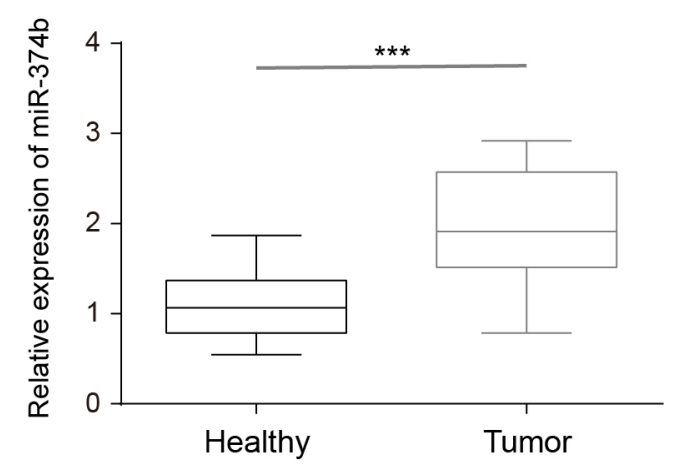

C

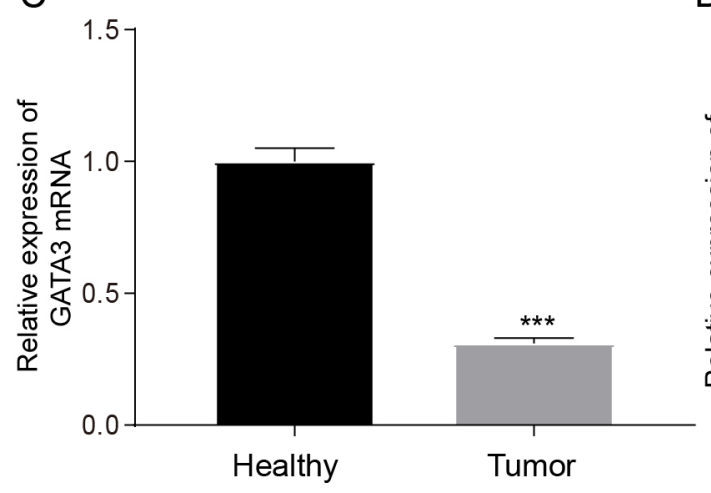

B

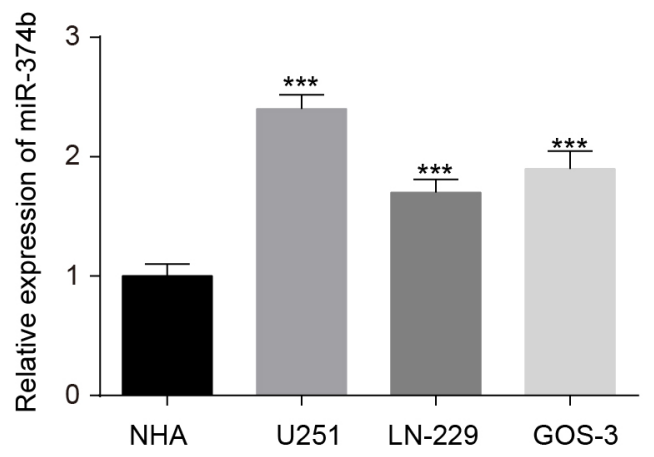

D

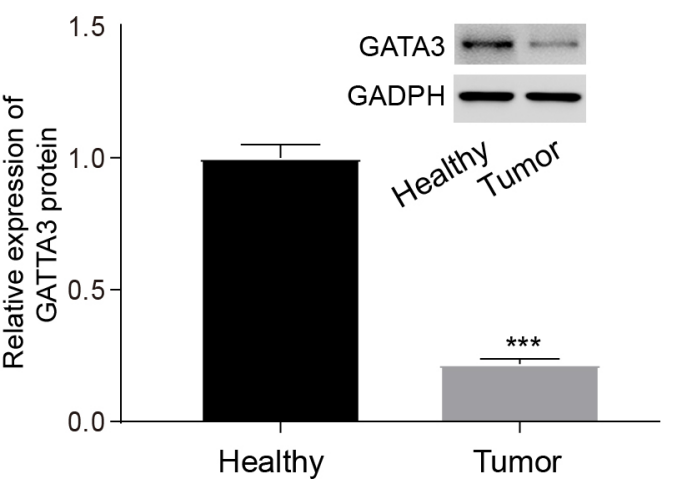

Figure 1. MiR-374b was upregulated in glioma and cell lines. A) qRT-PCR was employed to detect the expression of miR-374b in tumor and healthy tissues. B) The expression of miR-374b in three kinds of glioma cell lines, U251 that was chosen for further investigation in this research, LN-229 and GOS-3, and normal human astrocyte NHA were demonstrated by qRT-PCR. C-D) The expression of GATA3 mRNA and protein in healthy or patients' tissues was investigated by $\mathrm{qRT}$-PCR or $\mathrm{WB}$ assay, respectively. ${ }^{* *} \mathrm{P}<0.001$, compared with Healthy group or NHA group.

(Figures 2C-D). Because of the significance of tumor metastasis in the progression of glioma, cell migration ability was explored by transwell assay. The number of migrating cells in mimics group was higher than in NC group, which means more powerful metastatic ability (Figures 2E-F). Meanwhile, the results uncovered that inhibition of $\mathrm{miR}-374 \mathrm{~b}$ expression contributed to weaker cell migration ability than that in NC group (Figures 2E-F). These results hinted that miR-374b might promote tumorigenesis via elevating cell viability, cell migration ability and reducing apoptosis ratio.

MiR-374b targeted GATA3. According to the predicting results of TargetScan database (http://www.targetscan.org/ vert_72/), miR-374b might target GATA3 to influence the glioma progression. To validate the regulatory relationship between miR-374b and GATA3, dual luciferase assay was conducted. In the present study, glioma cells transfected with luciferase reporter plasmid recombining GATA3-wt or GATA3-mut sequence (Figure 3A) were co-transfected with $\mathrm{miR}-374 \mathrm{~b}$ mimics or the matched control sequence. As shown in Figure 3B, the relative luciferase expression of cells transfected with miR-374b mimics in GATA3-wt group was significantly inhibited compared with NC group. However, injection of miR-374b mimics did not change the luciferase viability of GATA3-wt group cells. qRT-PCR and western blot assay further proved this conclusion and results demonstrated that mimics transfection suppressed GATA3 mRNA and protein expression, but injecting inhibitors resulted in enhancement in GATA3 mRNA and protein expression (Figures 3C-D).

MiR-374b targeted GATA3 involvement in glioma. After proving miR-374b targeted GATA3, it was assumed that miR-374b promoted glioma cell migration and viability and hindered cell apoptosis via suppressing GATA3 as a tumor suppressor gene. To verify the hypothesis, si1-GATA3 and si2-GATA3 transfection was performed to knockdown GATA3. pc-GATA3 was used for GATA3 upregulation. As shown in Figure 4A, si1-GATA3 and si2-GATA3 could effectively inhibit GATA3 expression and pc-GATA3 also successfully elevated GATA3 mRNA level. Besides, the inhibition of si1-GATA3 in GATA3 expression was stronger than si2-GATA3, so si1-GATA3 was employed for further experiments. As shown in Figure 4B, sil-GATA3, pc-GATA3, mimics + pc-GATA3 and inhibitors + si1-GATA3 were transfected into U251 cells for deregulating GATA3. The 
A

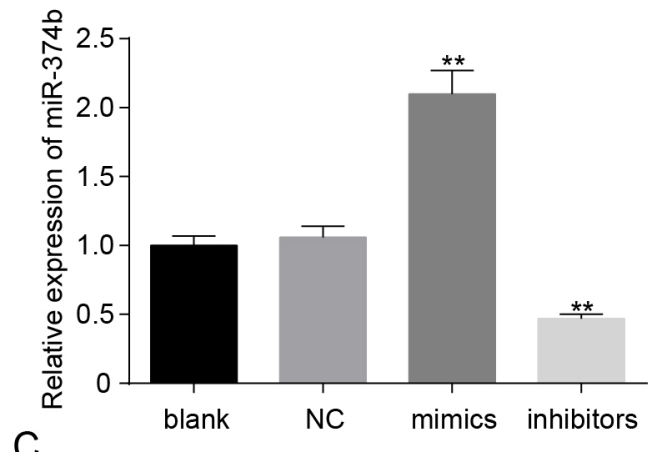

C
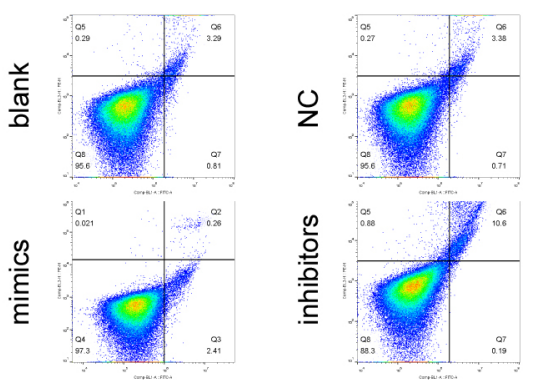

$\mathrm{E}$
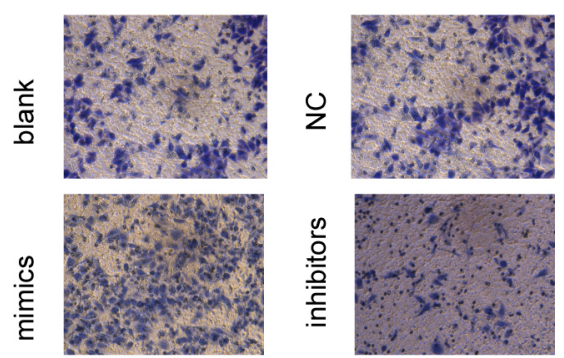

B
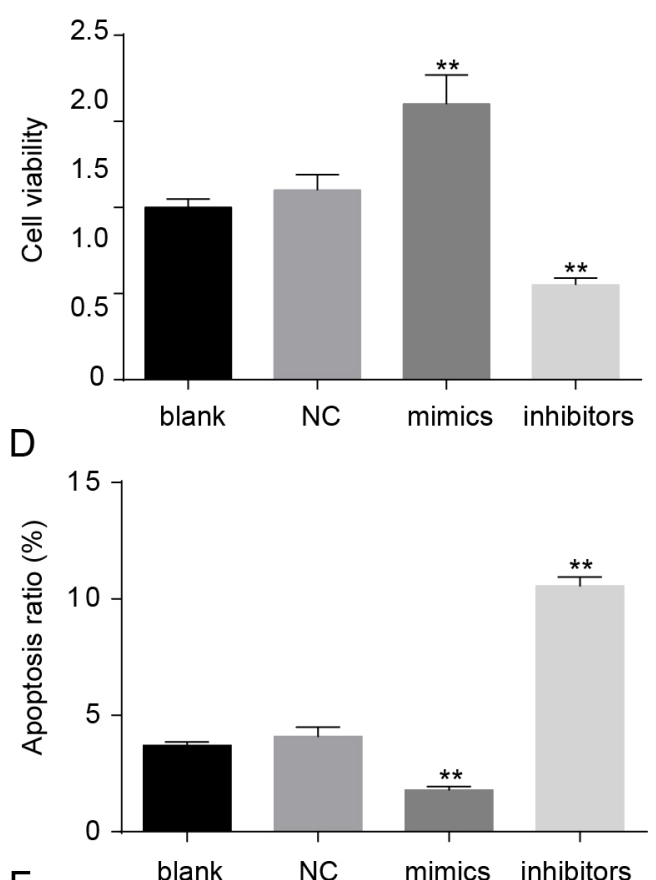

F

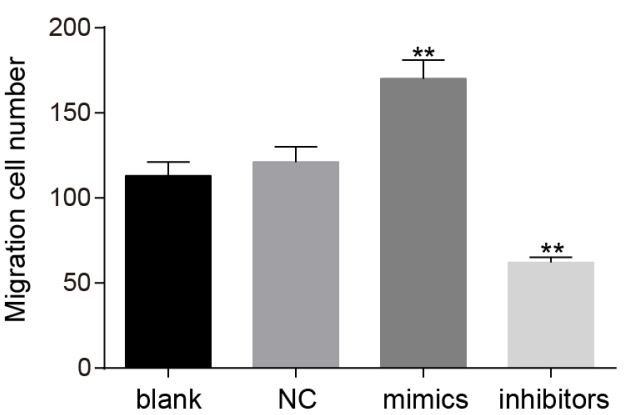

Figure 2. MiR-374b promoted glioma process in vitro. A) Mimics and inhibitors were used to regulate miR-374b expression that was determined by qRT-PCR assay in U251 cell line. B) Cell viability was investigated by CCK-8 assay after cells being transfected with NC, mimics and inhibitors or blank. C-D) Cell apoptosis was assessed by FCM assay after cell being transfected with NC, mimics and inhibitors or blank. E-F) Cell migration ability was demonstrated by transwell analysis in transfecting cells. ${ }^{* *} \mathrm{p}<0.01$, compared with NC group.

results suggested that mimics co-transfected with pc-GATA3 could reverse the increase in GATA3 expression promoted by pc-GATA3 and inhibitors + sil-GATA3 group showed lower GATA3 mRNA level than sil-GATA3 group. Afterwards, cell viability in each group was explored. Obviously, cell viability was enhanced by si-GATA3 compared with NC group but decreased by pc-GATA3. However, mimics + pc-GATA3 and inhibitors + si1-GATA3, respectively reversed the effectiveness of pc-GATA3 and sil-GATA3 (Figure 4C). On the contrary, sil-GATA3 decreased cell apoptosis ratio, which was opposite to the role of pc-GATA3. Mimics or inhibitors co-transfected with pc-GATA3 or si1-GATA3, respectively could attenuate the changes in apoptosis (Figure 4D). Moreover, cell migration was promoted by sil-GATA3 but inhibited by pc-GATA3 and in the same way mimics +
pc-GATA3 and inhibitors + si1-GATA3 reversed the function of pc-GATA3 and si1-GATA3, respectively (Figure 4E).

GATA3 binding to the promoter of SEMA3B. In the previous study, promotion of SEMA3B expression by GATA3 as transcription factor has been uncovered [27]. In our investigation, this regulatory relationship was evaluated. Figure $5 \mathrm{~A}$ indicated that when GATA3 was silenced, the input rate of SEMA3B promoter was lower comparing with the normal group, where the IgG was considered as positive control. Next, we altered GATA3 in U251 cells and monitored alterations in GATA3 and SEMA3B mRNA expression levels. Obviously, overexpression of GATA3 resulted in significant upregulation of SEMA3B, but GATA3silencing exhibited suppressive role in SEMA3B expression (Figure 5B). To get more credible evidence, the SEMA3B protein expression levels was also 

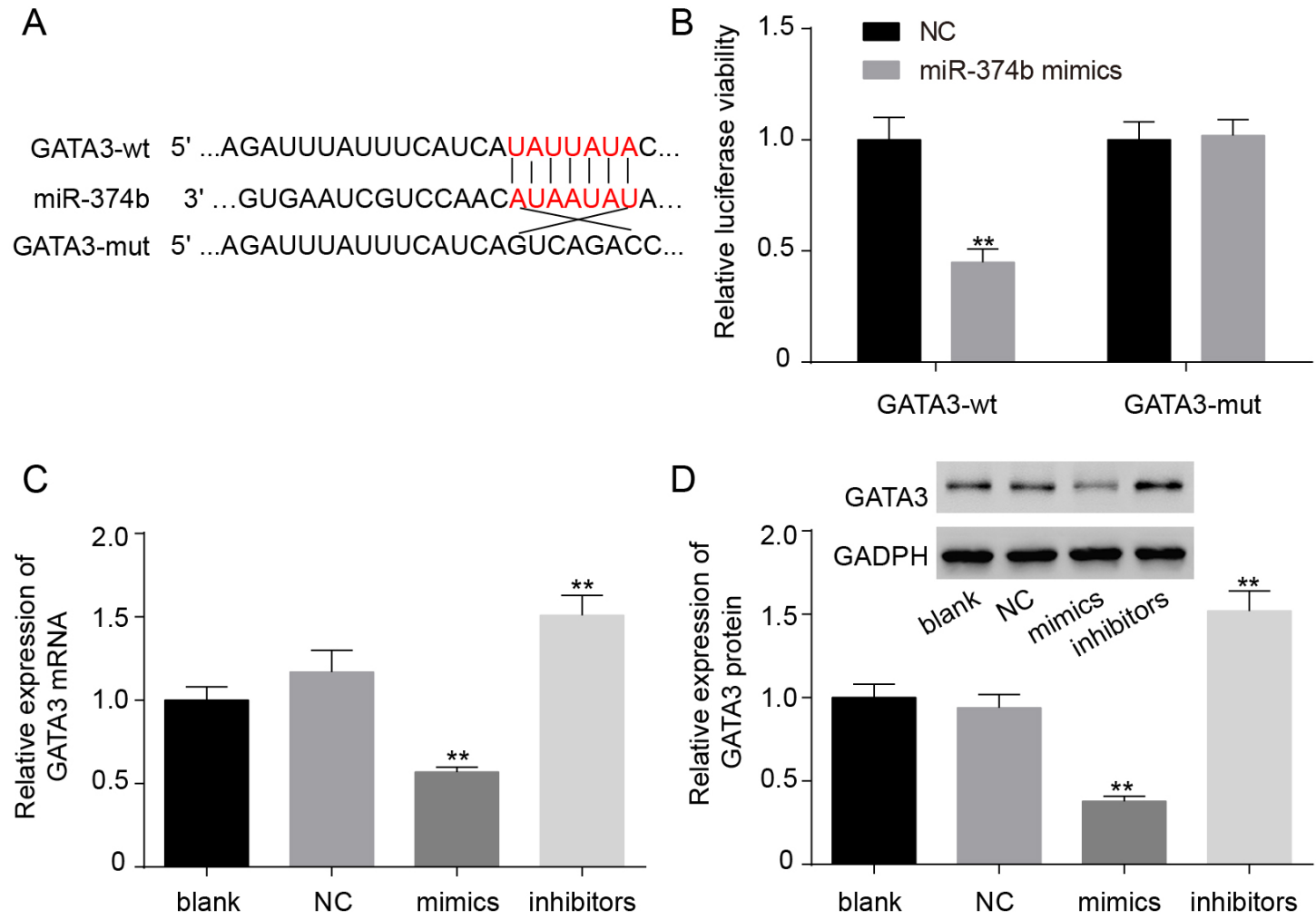

Figure 3. MiR-374b targeted GATA3. A) The targeting sequence of miR-374b and GATA3. B) Dual luciferase assay was utilized to analyze the effects of miR-374b on GATA3-wt and GATA3-mut. C-D) qRT-PCR and western blot assay were used to detect changes of GATA3 mRNA and protein after transfection. ${ }^{* *} \mathrm{p}<0.01$, compared with NC group.

monitored and the results were parallel to SEMA3B mRNA level (Figures 5C-D).

MiR-374b promoted glioma progression via targeting GATA3 to inhibit SEMA3B. To validate the influence of miR-374b on SEMA3B, pc-SEMA3B for up-regulating SEMA3B, mimics and mimics + pc-SEMA3B were transfected into U251 cells and then GATA3 along with SEMA3B mRNA and protein expressions were confirmed. From the results, it was noted that SEMA3B expression was positively correlated with GATA3 (Figures 6A-C). When cells were transfected with mimics, GATA3 mRNA and protein expressions were inhibited resulting in lower SEMA3B expression. However, transfecting pc-SEMA3B did not influence GATA3 but significantly elevated SEMA3B expression, meaning that GATA3 was not indispensable for SEMA3B expression. Furthermore, mimics + pc-SEMA3B transfection contributed to reverse the inhibition in SEMA3B resulting from mimics (Figures 6A-C). Next, the role of pc-SEMA3B, mimics and mimics + pc-SEMA3B on cell viability, apoptosis and migration have been elucidated. In Figure 6D, it was obvious that pc-SEMA3B significantly repressed cell viability and mimics promoted that. Besides, mimics co-transfected with pc-SEMA3B ameliorated the increase in cell viability induced by transfecting mimics. Meanwhile, up-regulation of
SEMA3B accelerated cell apoptosis that was opposite to the effects of mimics that could repress SEMA3B transcription via GATA3 (Figures 6E-F). Equally, the function of mimics, inhibiting the apoptosis, could be reversed by co-transfection with pc-SEMA3B (Figures 6E-F). In Figure 6G-H, we could observe lower cell migration numbers in pc-SEMA3B group but higher numbers in mimics group than that in $\mathrm{NC}$ group. Similarly, mimics + pc-SEMA3B group manifested weaker migration ability compared with mimics group. These results elucidated that miR-374b involved in the glioma progression through targeting GATA3 that functioned as transcription factor via binding to the promoter of SEMA3B.

\section{Discussion}

In the present study, numerous experiments were conducted to investigate the particular molecular mechanism of miR-374b/GATA3/SEMA3B axis involved in progression and development of glioma and the results indicated that miR-374b exerted its function through mediating SEMA3B via targeting GATA3.

Numerous studies focused on pathogenesis and progress of tumors investigated the role of miRNAs. Wu et al. revealed that miR-192, regulating EGR1 and HOXB9, had potent 


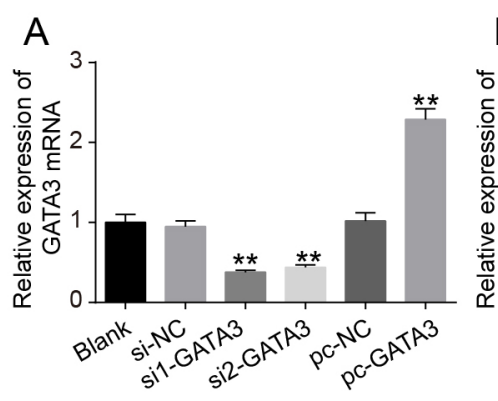

B

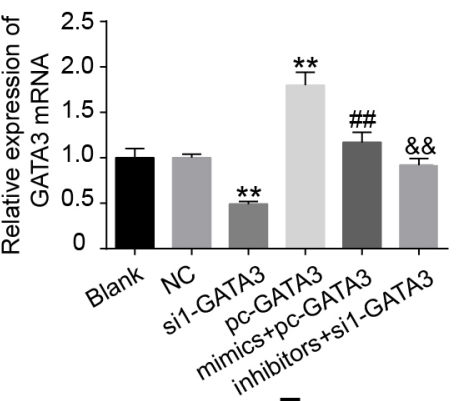

C

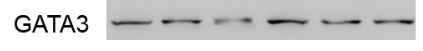

GADPH

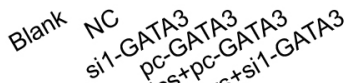

E

D
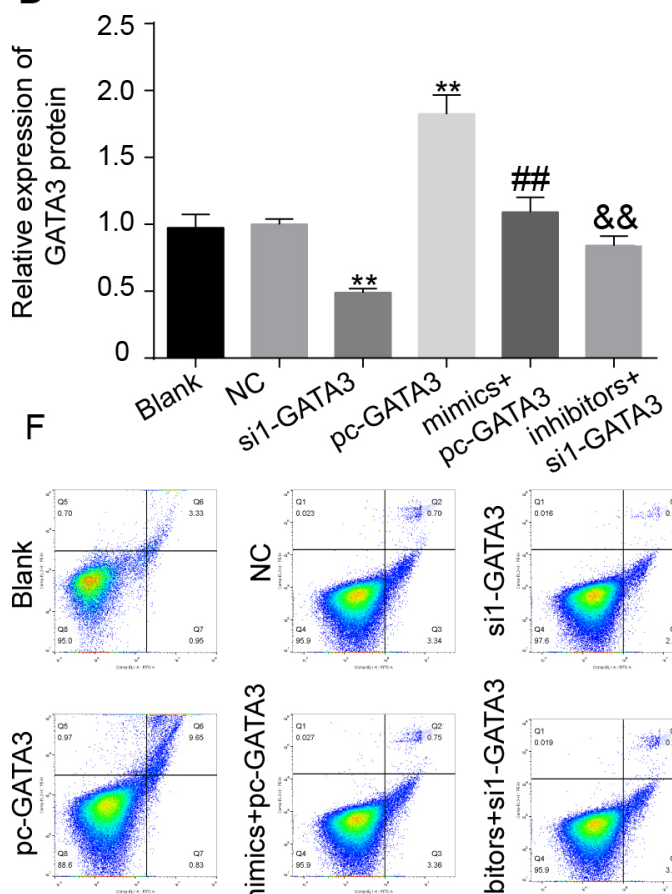

G

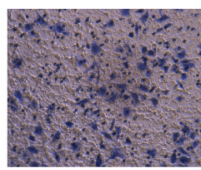

Blank

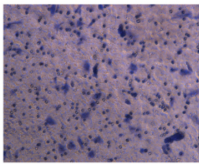

$p^{g}$ GRt
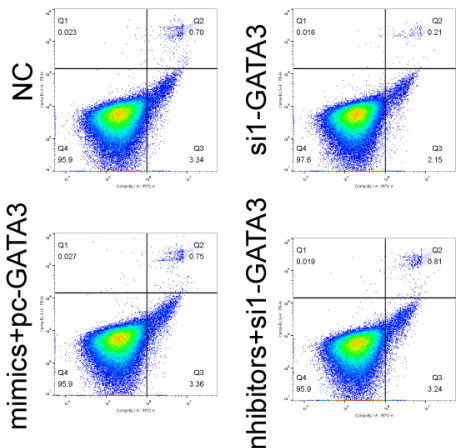
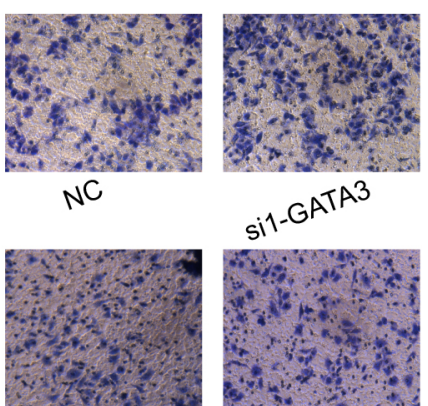

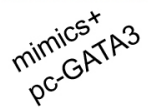

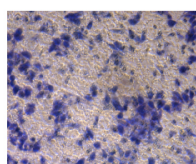

NC

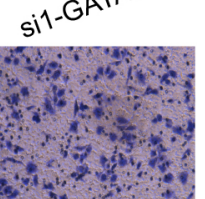

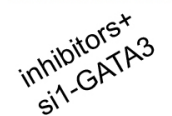
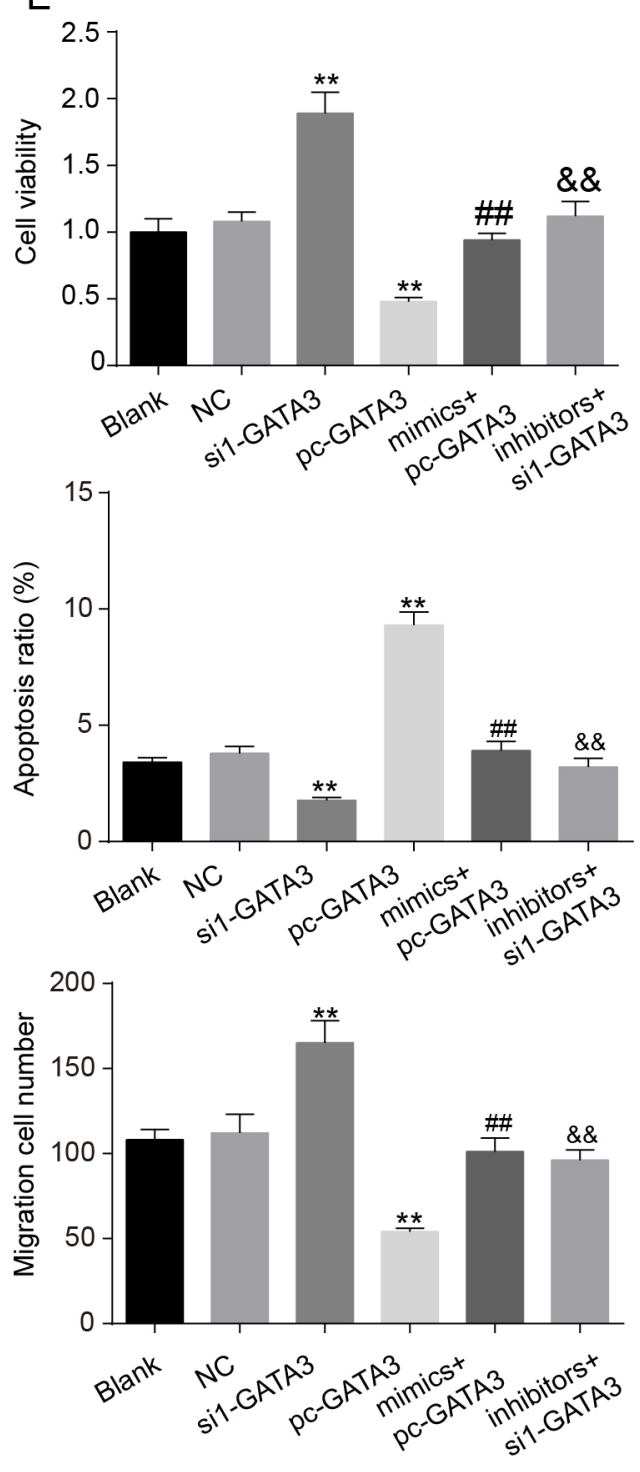

Figure 4. GATA3 suppressed glioma process and was targeted by miR-374b in vitro. A) qRT-PCR was used to determine GATA3 mRNA expression after si-NC, si1-GATA3, si2-GATA3, pc-NC and pc-GATA3 transfection. B) qRT-PCR was used to determine GATA3 mRNA expression after NC, si1GATA3, pc-GATA3, mimics + pc-GATA3 and inhibitors + si1-GATA3 transfection. C-D) GATA3 protein expression level was detected via WB assay after NC, si1-GATA3, pc-GATA3, mimics + pc-GATA3 and inhibitors + si1-GATA3 transfection. E) Cell viability was assessed by CCK-8 after NC, si1GATA3, pc-GATA3, mimics + pc-GATA3 and inhibitors + si1-GATA3 transfection. F) Apoptosis ratio was demonstrated after cell being transfected. G) Cell migration was measured by transwell assay. ${ }^{\star *} \mathrm{p}<0.01$, compared with NC group, \#\#p $<0.01$, compared with pc-GATA3 group, and $\& \& p<0.01$, compared with si1-GATA3 group. 

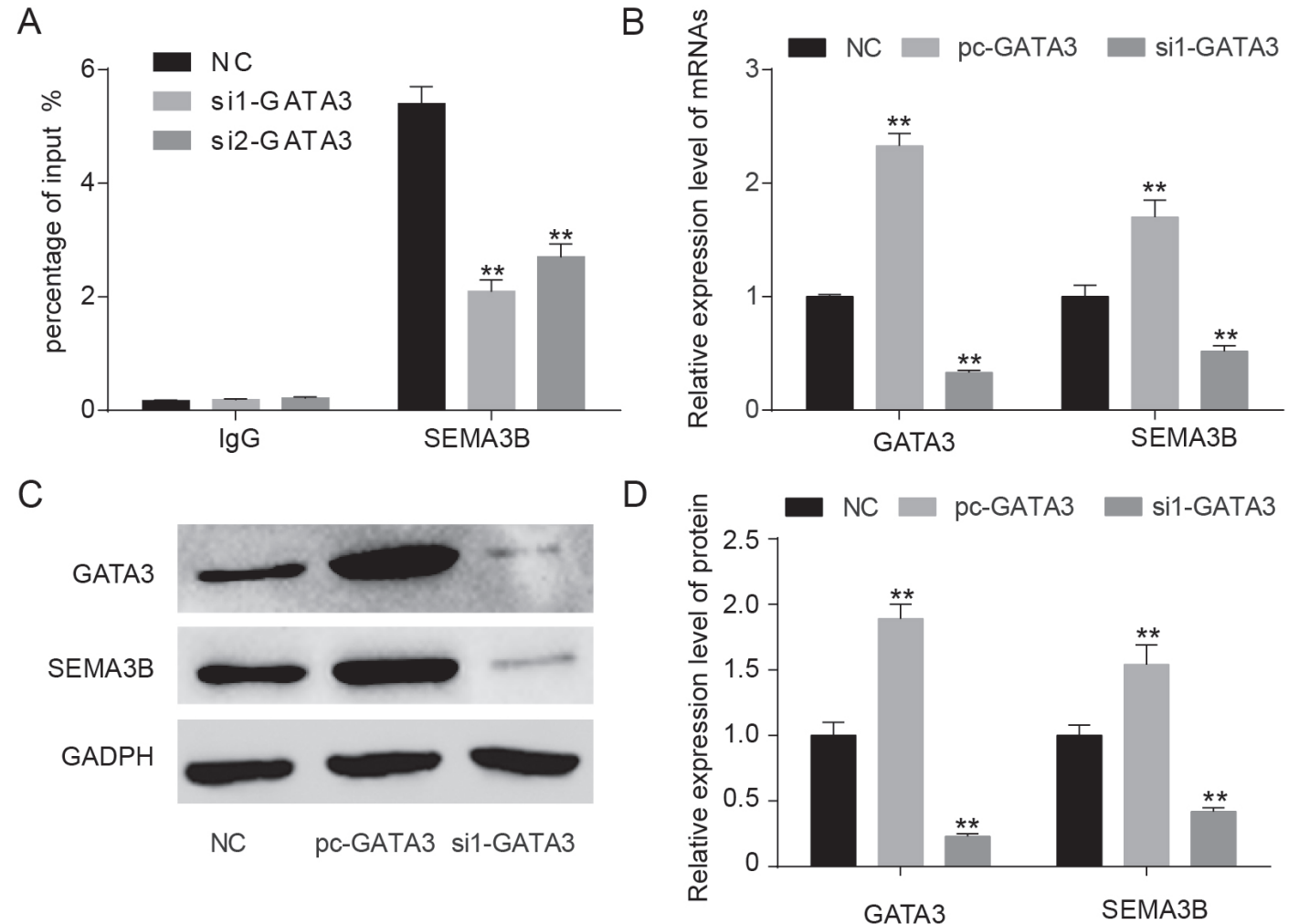

Figure 5. GATA3 bound to the promoter of SEMA3B. A) Chromatin immunoprecipitation was performed to identify the relationship between GATA3 and SEMA3B. B-D) After changing the GATA3 expression, SEMA3B mRNA and proteins expression level was assessed by qRT-PCR and western blot, respectively.

anti-angiogenic effect in several cancer types [30]. Besides, there was study that uncovered miR-31 could suppress Wnt signaling pathway to contribute to breast tumorigenesis [31]. Moreover, Sumazin et al. elucidated that massive miRNAs played crucial role in canonical oncogenic pathways in glioblastoma via mediating key genes, such as PTEN, PDGFRA and RUNX1 [32]. According to previous report, reversion inducing cysteine rich protein with kazal motifs (RECK) was a target gene of miR-374b, by which miR-374b significantly promoted gastric cancer cell invasion and metastasis [13]. Therefore, we analyzed miR-374b expression in patients with glioma and three glioma cell lines in comparison with normal tissues and cell lines. Our results suggested that miR-374b was upregulated in patients with glioma and tumor cell lines. However, the particular effects of miR-374b on pathogenesis and progress of glioma were indistinct.

To obtain the function of miR-374b in glioma, mimics and inhibitors have been employed and then the cell viability, apoptosis and migration were identified, results of which exhibited the promotion of miR-374b in glioma. Similarly, there was report indicating that miR-374b promoted proliferation and inhibited apoptosis through PI3K/Akt pathway in gastrointestinal stromal tumors [33]. Molecular classifica- tion of the mechanism of miR-374b involvement in glioma cell physiological process was helpful for clinical diagnosis and treatment. So, we predicted the target gene of miR-374b in glioma through TargetScan database, finding that GATA3 was a potential target gene of miR-374b (Figure $3 \mathrm{~A}$ ) as verified by dual luciferase assay. Substantial studies demonstrated tumor suppressor role of GATA3 in various cancers including bladder cancer [19], breast cancer [34] and glioblastoma [17]. In study of Pors et al., GATA3 showed obvious role in identifying mesonephric and mesonephric-like carcinomas of the gynecologic tract [40]. However, Yang et al. indicated that deficiency of GATA3-positive macrophages improves cardiac function in the patients with myocardial infarction or pressure overload hypertrophy [41]. Since the function of GATA3 and its relationship with miR-374b was vague, it deserved to be further investigated. In our study, upregulation GATA3 inhibited cell viability; migration but promoted apoptosis and miR-374b reversed the function of GATA3 in these issues. In the research of Zhong et al. [35], miR-720 targeting GATA3 was revealed. Homoplastically, Yang et al. elucidated that miR-200 inhibited expression of GATA3 [36]. Therefore, miR-374b might target GATA3 to suppress its expression thus contributing to pathogenesis and progress of glioma. 
A

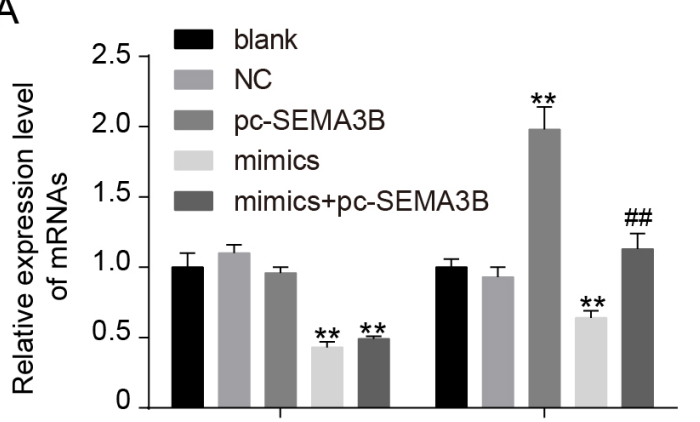

C

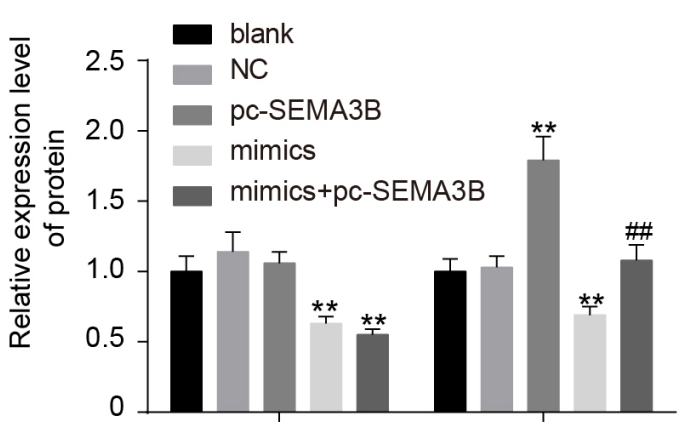

E

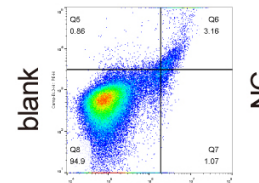

GATA3
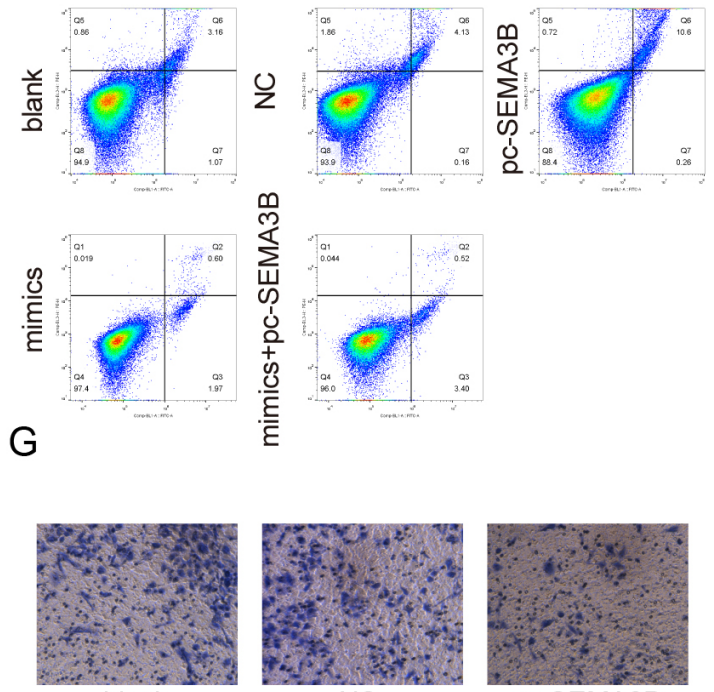

blank

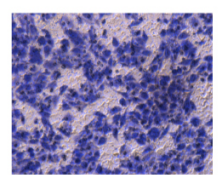

mimics
NC

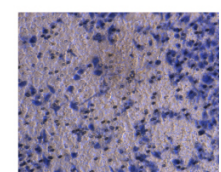

mimics+pc-SEMA3B

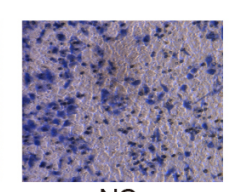

B

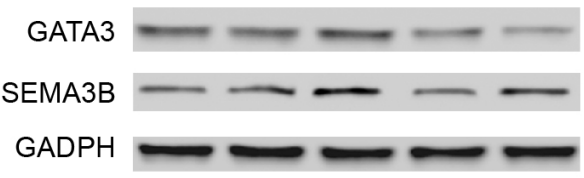

blank $N C$ SEMA3B mimiCS
mCSEA3B
mimicS

D

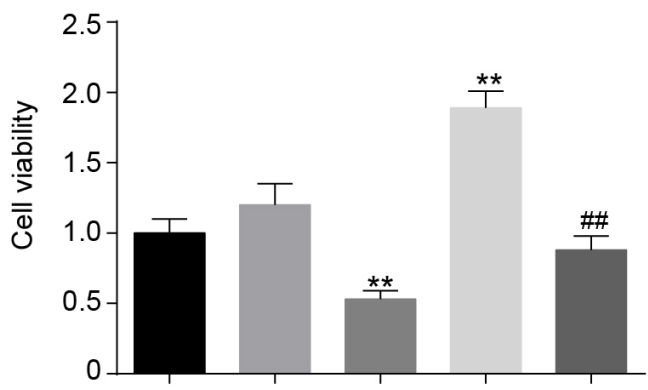

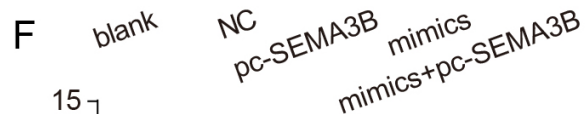
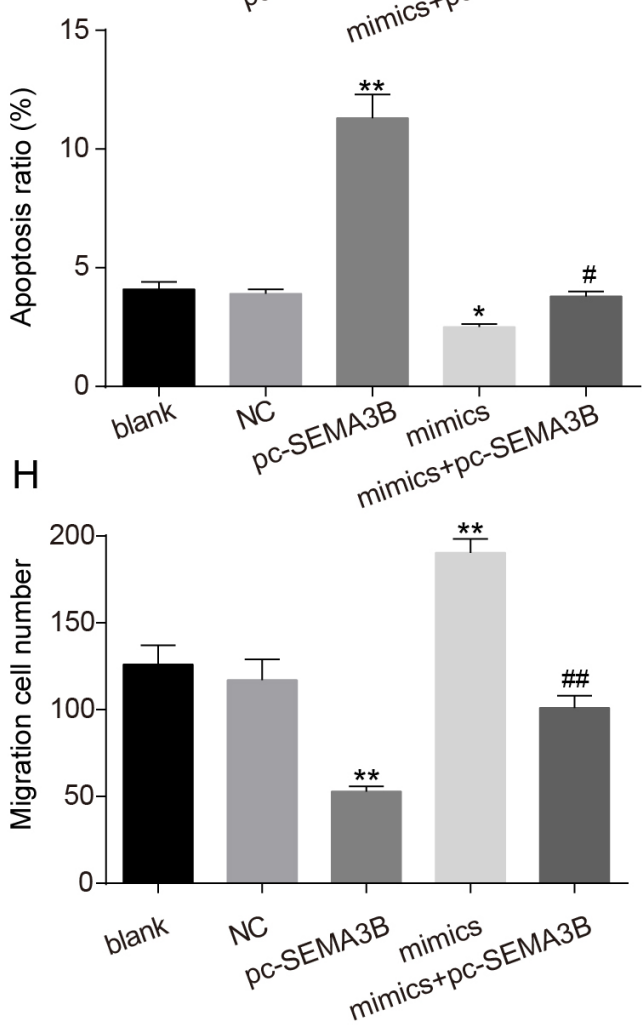

Figure 6. MiR-374b involved in the progression of glioma through inhibiting SEMA3B via targeting GATA3. A) The expression level of GATA3 mRNA and SEMA3B was identified after NC, pc-SEMA3B, mimics and mimics + pc-SEMA3B transfection. B-C) The expression level of GATA3 protein and SEMA3B was identified after NC, pc-SEMA3B, mimics and mimics + pc-SEMA3B transfection. D) Cell viability was assessed by CCK-8 after NC, pc-SEMA3B, mimics and mimics + pc-SEMA3B transfection. E-F) Apoptosis ratio was demonstrated after NC, pc-SEMA3B, mimics and mimics + pc-SEMA3B transfection. G-H) Cell migration was also measured by transwell assay. 
Semaphorin 3B (SEMA3B), as one of semaphorin (SEMA) superfamily, was a secreted axonal guidance molecule that contained a highly conserved and was expressed during development and throughout adulthood. According to Ryynanen et al., regulation SEMA expression level could affect the development of osteoblasts [42]. Moreover, there is report that SEMA3B and SEMA3G expression would disrupt neural network formation in the mammalian nervous system [43]. As reported by Shahi et al., SEMA3B was regulated by GATA3 that bound to the site of the promoter of SEMA3B [27]. However, identification of SEMA3B effect exerted in glioblastoma is weak. Hence in view of this, we assumed that miR-374b is involved in glioma progress by influencing SEMA3B via targeting GATA3. Numerous studies had identified that SEMA3B functioned as a tumor suppressor in various cancers, including non-small cell lung cancer [37], endometrial cancer [38] and glioblastoma [39]. In our study, enhancing the expression ofSEMA3B contributed to inhibition of cell migration and viability and acceleration of apoptosis.

Our results demonstrated that miR-374b post-transcriptionally hindered GATA3 expression resulting in attenuating SEMA3B expression and thus promoted invasion and metastasis of glioma cells. In the future, more studies should be conducted for exploring the role of miR/GATA3/SEMA3B axis in animal models of glioma and revealing more comprehensive relative mechanism.

In conclusion, this study discovered that human glioma cells had significant upregulation of miR-374b. Furthermore, miR-374b could facilitate migration and viability but decelerated apoptosis of glioma cells, possibly through suppressing SEMA3B via targeting GATA3. Our findings introduced novel biomarkers that could potentially provide new therapeutic strategies for glioma.

Acknowledgements: This work was supported by Inner Mongolia Science \& Technology Plan; The project YKD2016KJBW002 is supported by Inner Mongolia Medical University Science \& Technology Billion Program; The project NJZY17115 is supported by Inner Mongolia Autonomous Region University \& College Science \& Technology Program; The project ZYFYZD014 is supported by Inner Mongolia Medical University Affiliated Hospital Primary Program; The project 201702081 is supported by Inner Mongolia Autonomous Region Health \& Family Planning Committee Science \& Technology Program.

\section{References}

[1] SRINIVASAN VM, FERGUSON SD, LEE S, WEATHERS SP, KERRIGAN BCP et al. Tumor Vaccines for Malignant Gliomas. Neurotherapeutics 2017; 14: 345-357. https://doi. org/10.1007/s13311-017-0522-2

[2] OSTROM QT, GITTLEMAN H, LIAO P, ROUSE C, CHEN $Y$ et al. CBTRUS statistical report: primary brain and central nervous system tumors diagnosed in the United States in 2007-2011. Neuro Oncol 2014; 16 Suppl 4: iv1-63. https:// doi.org/10.1093/neuonc/nou223
[3] OMURO A, DEANGELIS LM. Glioblastoma and other malignant gliomas: a clinical review. JAMA 2013; 310: 1842 1850. https://doi.org/10.1001/jama.2013.280319

[4] STUPP R, WONG ET, KANNER AA, STEINBERG D, ENGELHARD $\mathrm{H}$ et al. NovoTTF-100A versus physician's choice chemotherapy in recurrent glioblastoma: a randomised phase III trial of a novel treatment modality. Eur J Cancer 2012; 48: 2192-2202. https://doi.org/10.1016/j. ejca.2012.04.011

[5] RICARD D, IDBAIH A, DUCRAY F, LAHUTTE M, HOANG-XUAN K et al. Primary brain tumours in adults. Lancet 2012; 379: 1984-1996. https://doi.org/10.1016/S01406736(11)61346-9

[6] SHI Z, CHEN Q, LI C, WANG L, QIAN X et al. MiR-124 governs glioma growth and angiogenesis and enhances chemosensitivity by targeting R-Ras and N-Ras. Neuro Oncol 2014; 16: 1341-1353. https://doi.org/10.1093/neuonc/ nou084

[7] KHODZITSKAIA VK, ZOSIMOV AN. [Specific risk factors of the development of early period of primary tuberculous infection in children]. Probl Tuberk 1994; 5: 7-9.

[8] WANG JX, ZHANG XJ, LI Q, WANG K, WANG Y et al. MicroRNA-103/107 Regulate Programmed Necrosis and Myocardial Ischemia/Reperfusion Injury Through Targeting FADD. Circ Res 2015; 117: 352-363. https://doi.org/10.1161/ CIRCRESAHA.117.305781

[9] LONG JM, LAHIRI DK. Advances in microRNA experimental approaches to study physiological regulation of gene products implicated in CNS disorders. Exp Neurol 2012; 235: 402-418. https://doi.org/10.1016/j.expneurol.2011.12.043

[10] BARTEL DP. MicroRNAs: target recognition and regulatory functions. Cell 2009; 136: 215-233. https://doi.org/10.1016/j. cell.2009.01.002

[11] GUO M, ZHANG X, WANG G, SUN J, JIANG Z et al. miR603 promotes glioma cell growth via Wnt/beta-catenin pathway by inhibiting WIF1 and CTNNBIP1. Cancer Lett 2015; 360: 76-86. https://doi.org/10.1016/j.canlet.2015.02.003

[12] XU W, LIU M, PENG X, ZHOU P, ZHOU J et al. miR-24$3 \mathrm{p}$ and miR-27a-3p promote cell proliferation in glioma cells via cooperative regulation of MXI1. Int J Oncol 2013; 42: 757-766. https://doi.org/10.3892/ijo.2012.1742

[13] XIE J, TAN ZH, TANG X, MO MS, LIU YP et al. MiR374b-5p suppresses RECK expression and promotes gastric cancer cell invasion and metastasis. World J Gastroenterol 2014; 20: 17439-17447. https://doi.org/10.3748/wjg.v20. i46.17439

[14] KOUROS-MEHR H, SLORACH EM, STERNLICHT MD, WERB Z. GATA-3 maintains the differentiation of the luminal cell fate in the mammary gland. Cell 2006; 127: 10411055. https://doi.org/10.1016/j.cell.2006.09.048

[15] CANCER GENOME ATLAS NETWORK. Comprehensive molecular portraits of human breast tumours. Nature 2012; 490: 61-70. https://doi.org/10.1038/nature11412

[16] JIANG YZ, YU KD, ZUO WJ, PENG WT, SHAO ZM. GATA3 mutations define a unique subtype of luminal-like breast cancer with improved survival. Cancer 2014; 120: 1329-1337. https://doi.org/10.1002/cncr.28566 
[17] MAJEWSKA E, ROLA R, BARCZEWSKA M, MARQUEZ J, ALBRECHT J et al. Transcription factor GATA3 expression is induced by GLS2 overexpression in a glioblastoma cell line but is GLS2-independent in patient-derived glioblastoma. J Physiol Pharmacol 2017; 68: 209-214.

[18] LI B, JIN X, MENG H, HU B, ZHANG T et al. Morin promotes prostate cancer cells chemosensitivity to paclitaxel through miR-155/GATA3 axis. Oncotarget 2017; 8: 4784947860. https://doi.org/10.18632/oncotarget.18133

[19] LI Y, ISHIGURO H, KAWAHARA T, KASHIWAGI E, IZUMI K et al. Loss of GATA3 in bladder cancer promotes cell migration and invasion. Cancer Biol Ther 2014; 15: 428-435. https://doi.org/10.4161/cbt.27631

[20] SABAG AD, SMOLKIN T, MUMBLAT Y, UEFFING M, KESSLER $O$ et al. The role of the plexin-A2 receptor in Sema3A and Sema3B signal transduction. J Cell Sci 2014; 127: 5240-5252. https://doi.org/10.1242/jcs.155960

[21] CHEN R, ZHUGE X, HUANG Z, LU D, YE X et al. Analysis of SEMA3B methylation and expression patterns in gastric cancer tissue and cell lines. Oncol Rep 2014; 31: 1211-1218. https://doi.org/10.3892/or.2014.2972

[22] CASTRO-RIVERA E, RAN S, THORPE P, MINNA JD. Semaphorin 3B (SEMA3B) induces apoptosis in lung and breast cancer, whereas VEGF165 antagonizes this effect. Proc Natl Acad Sci U S A 2004; 101: 11432-11437. https:// doi.org/10.1073/pnas.0403969101

[23] LERMAN MI, MINNA JD. The 630-kb lung cancer homozygous deletion region on human chromosome 3 p21.3: identification and evaluation of the resident candidate tumor suppressor genes. The International Lung Cancer Chromosome 3p21.3 Tumor Suppressor Gene Consortium. Cancer Res 2000: 60: 6116-6133.

[24] SOKER S, FIDDER H, NEUFELD G, KLAGSBRUN M. Characterization of novel vascular endothelial growth factor (VEGF) receptors on tumor cells that bind VEGF165 via its exon 7-encoded domain. J Biol Chem 1996: 271: 5761-5767.

[25] MAESTRINI E, TAMAGNONE L, LONGATI P, CREMONA O, GULISANO $\mathrm{M}$ et al. A family of transmembrane proteins with homology to the MET-hepatocyte growth factor receptor. Proc Natl Acad Sci U S A 1996; 93: 674678.

[26] OHTA K, MIZUTANI A, KAWAKAMI A, MURAKAMI Y, KASUYA Y et al. Plexin: a novel neuronal cell surface molecule that mediates cell adhesion via a homophilic binding mechanism in the presence of calcium ions. Neuron 1995; 14: 1189-1199.

[27] SHAHI P, WANG CY, CHOU J, HAGERLING C, GONZALEZ VELOZO H et al. GATA3 targets semaphorin 3B in mammary epithelial cells to suppress breast cancer progression and metastasis. Oncogene 2017; 36: 5567-5575. https:// doi.org/10.1038/onc.2017.165

[28] RICH JN, HANS C, JONES B, IVERSEN ES, MCLENDON $\mathrm{RE}$ et al. Gene expression profiling and genetic markers in glioblastoma survival. Cancer Res 2005; 65: 4051-4058. https://doi.org/10.1158/0008-5472.CAN-04-3936
[29] KARAYAN-TAPON L, WAGER M, GUILHOT J, LEVILLAIN P, MARQUANT C et al. Semaphorin, neuropilin and VEGF expression in glial tumours: SEMA3G, a prognostic marker? Br J Cancer 2008; 99: 1153-1160. https://doi. org/10.1038/sj.bjc.6604641

[30] WU SY, RUPAIMOOLE R, SHEN F, PRADEEP S, PECOT CV et al. A miR-192-EGR1-HOXB9 regulatory network controls the angiogenic switch in cancer. Nat Commun 2016; 7: 11169. https://doi.org/10.1038/ncomms11169

[31] LV C, LI F, LI X, TIAN Y, ZHANG Y et al. MiR-31 promotes mammary stem cell expansion and breast tumorigenesis by suppressing Wnt signaling antagonists. Nat Commun 2017; 8: 1036. https://doi.org/10.1038/s41467-017-01059-5

[32] SUMAZIN P, YANG X, CHIU HS, CHUNG WJ, IYER A et al. An extensive microRNA-mediated network of RNARNA interactions regulates established oncogenic pathways in glioblastoma. Cell 2011; 147: 370-381. https://doi. org/10.1016/j.cell.2011.09.041

[33] LONG ZW, WU JH, CAI H, WANG YN, ZHOU Y. MiR374b Promotes Proliferation and Inhibits Apoptosis of Human GIST Cells by Inhibiting PTEN through Activation of the PI3K/Akt Pathway. Mol Cells 2018; 41: 532-544. https:// doi.org/10.14348/molcells.2018.2211

[34] SI W, HUANG W, ZHENG Y, YANG Y, LIU X et al. Dysfunction of the Reciprocal Feedback Loop between GATA3and ZEB2-Nucleated Repression Programs Contributes to Breast Cancer Metastasis. Cancer Cell 2015; 27: 822-836. https://doi.org/10.1016/j.ccell.2015.04.011

[35] ZHONG Y, YI C. MicroRNA-720 suppresses M2 macrophage polarization by targeting GATA3. Biosci Rep 2016; 36 . https://doi.org/10.1042/BSR20160105

[36] YANG Y, AHN YH, GIBBONS DL, ZANG Y, LIN W et al. The Notch ligand Jagged2 promotes lung adenocarcinoma metastasis through a miR-200-dependent pathway in mice. J Clin Invest 2011; 121: 1373-1385. https://doi.org/10.1172/ JCI42579

[37] ITO M, ITO G, KONDO M, UCHIYAMA M, FUKUI T et al. Frequent inactivation of RASSF1A, BLU, and SEMA3B on 3 p21.3 by promoter hypermethylation and allele loss in non-small cell lung cancer. Cancer Lett 2005; 225: 131-139. https://doi.org/10.1016/j.canlet.2004.10.041

[38] NGUYEN H, IVANOVA VS, KAVANDI L, RODRIGUEZ GC, MAXWELL GL et al. Progesterone and 1,25-dihydroxyvitamin $\mathrm{D}(3)$ inhibit endometrial cancer cell growth by upregulating semaphorin $3 \mathrm{~B}$ and semaphorin 3F. Mol Cancer Res 2011; 9: 1479-1492. https://doi.org/10.1158/15417786.MCR-11-0213

[39] OCHI K, MORI T, TOYAMA Y, NAKAMURA Y, ARAKAWA H. Identification of semaphorin3B as a direct target of p53. Neoplasia 2002; 4: 82-87. https://doi.org/10.1038/sj/ neo/7900211

[40] PORS J, CHENG A, LEO JM, KINLOCH M. A, GILKS B et al. A Comparison of GATA3, TTF1, CD10, and Calretinin in Identifying Mesonephric and Mesonephric-like Carcinomas of the Gynecologic Tract. Am J Surg Pathol 2018; 12: 1596-1606. https://doi.org/10.1097/PAS.0000000000001142 
[41] YANG M, SONG L, WANG L, YUKHT A, RUTHER H et al. Deficiency of GATA3-Positive Macrophages Improves Cardiac Function Following Myocardial Infarction or Pressure Overload Hypertrophy. J Am Coll Cardiol 2018; 8: 885-904. https://doi.org/10.1016/j.jacc.2018.05.061

[42] RYYNANEN J, KRIEBITZSCH C, MEYER MB, JANSSENS I, PIKE JW et al. Class 3 semaphorins are transcriptionally regulated by $1,25(\mathrm{OH}) 2 \mathrm{D} 3$ in osteoblasts. J Steroid Biochem Mol Biol 2018; 173: 185-193. https://doi.org/10.1016/j.jsbmb.2017.02.005
[43] KIMURA E, ENDO T, YOSHIOKA W, DING Y, UJITA W et al. In utero and lactational dioxin exposure induces Sema3b and Sema3g gene expression in the developing mouse brain. Biochem Biophys Res Commun 2016; 476: 108-113. https:// doi.org/10.1016/j.bbrc.2016.05.048 